Article

\title{
Monin-Obukhov similarity theory for modeling of wind turbine wakes under atmospheric stable conditions: breakdown and modifications
}

\author{
Xing Xing Han ${ }^{1}{ }^{\oplus}$, De You Liu ${ }^{1}$, Chang $\mathrm{Xu}^{2, *}$, Wen Zhong Shen ${ }^{3}$, Lin Min $\mathrm{Li}^{2}$ and Fei Fei Xue ${ }^{1}$ \\ 1 College of Water Conservancy and Hydropower Engineering, Hohai University, China; hantone@hhu.edu.cn \\ (Xing Xing Han), Liudyhhuc@163.com (De You Liu), xuefeifeihhu@163.com(Fei Fei Xue) \\ 2 College of Energy and Electrical Engineering, Hohai University, China; lilinmin@hhu.edu.cn (Lin Min Li) \\ 3 Department of Wind Energy, Technical University of Denmark, Denmark; wzsh@dtu.dk \\ * Correspondence: zhuifengxu@hhu.edu.cn;
}

\begin{abstract}
Monin-Obukhov similarity theory (MOST) overestimates wind shear in some atmospheric stable conditions, i.e. Richardson number $R_{f}<0.25$. The overestimated wind shear that leads to an under-predicted friction wind speed and a lower ambient turbulence intensity for a given hub-height reference wind speed and a given roughness length, could influence wake modeling of a wind turbine. This work investigates the side effects of the breakdown of MOST on wake modeling under stable conditions and makes some modifications to the flow similarity functions to eliminate these side effects. Based on a field measurement in a wind farm, we firstly show that MOST predicts a larger wind shear for the atmospheric stability parameter $\zeta>0.1$ and proposes new flow similarity functions without constraining $R_{f}$ to limit the overestimated wind shear by MOST. Next, different turbulence models based on MOST and a modified one based on the new similarity functions are investigated through numerical simulations. These turbulence models are combined with the actuator disk model (AD) and Reynolds-averaged Navier-Stokes equations (RANS) to model wind turbine wakes under stable conditions. As compared to measurements, numerical results show that turbulence models based on MOST result in larger wake deficits and slower wake recovery rate with a square root of the mean-squared-error (RSME) of wake deficit in the range of 0.07-0.18. This overestimated wake effect is improved by applying the new similarity functions and the RSME of wake deficit is averagely reduced by 0.05 . Finally, we check the role of the under-predicted turbulence intensity playing in the larger wake deficit predicted by models based MOST. Additional numerical simulations using the modified turbulence model are carried out, in which the roughness length is reduced to impose a hub-height ambient turbulence intensity equivalent to the MOST case. Simulation results show that reducing turbulence intensity enhances wake effects, however, it cannot reproduce the large wake deficit predicted by models based on MOST, which suggests that the overestimated wake effect by MOST could be also related to the overestimated wind shear.
\end{abstract}

Keywords: wind turbine; wake; atmospheric stability; MOST; turbulence models

\section{Introduction}

As a measure of turbulence exchanges in the atmospheric surface layer, atmospheric stability can significantly affect the wind turbine wake deficit and its recovery rate. In general, turbulence exchanges between the wake and the atmosphere are depressed under stable conditions. High wake deficits and slow wake recovery thus are usually observed in the stable stratification boundary layer $[1,2]$. Since wakes play critical roles in wind farm energy production and the fatigue loads of wind 
turbines, there is an increasing interest in studying the effects of atmospheric stability on wakes and developing wake models for non-neutral conditions.

The impact of atmospheric stability on wakes are widely observed in wind tunnel measurements of small-scale models of wind turbines [3-5] and full-scale field experiments [1,2,6-8]. According to the wind tunnel measurements in Chamorror et al. [3], the stronger inlet wind shear in the stable case leads to a slightly stronger turbulence intensity and extends the region of enhanced turbulence intensity from a distance of about $4-5.5$ rotor diameters to 3 and 6 rotor diameters downwind of the turbine location. In Zhang et al. [4], a 15\% smaller velocity deficit at the wake center, a more rapid momentum recovery due to an enhanced radial momentum transport, a $20 \%$ higher peak turbulence intensity were observed in the unstable case, as compared to the wake of the same wind turbine under neutral conditions. According to the field experiments of wakes using LiDARs or masts, Magnusson et al. [6], Iungo et al. [7] and Han et al. [2] also found that the velocity deficit decreases more slowly under stable conditions and more quickly under unstable conditions. Those observations suggest that atmospheric stability should be considered for improved wake models and predictions of wind power harvesting.

Besides field measurements and wind tunnel experiments of wind turbine wakes under non-neutral conditions, numerical models based on the computational fluid dynamics (CFD) have also been used to investigate the effect of atmospheric stability on the wind turbine wakes [1,9-14] .High-fidelity large eddy simulations with the actuator line model (AL-LES) $[9,10]$ or the actuator disk model (AD-LES) $[1,11]$ are commonly used to study the structure and dynamics of wind turbine wakes in varying stability cases. This is mainly because that the LES approaches allow to capture the near wake structure, resolve the interactions of tip vortices with large-scale eddies of the ambient flow and wake meandering. However, the high-fidelity approaches are computationally expensive for wind energy engineering applications. There are some efforts towards developing turbulence models for wind turbine wakes under non-neutral conditions in RANS to reduce computational costs.

A widely used turbulence model for the thermal stratified boundary layer was developed by Alinot and Masson [15] where a coefficient of the buoyant terms in the $\varepsilon$ transport equation is calibrated with atmospheric stability. However, van der Laan et al. [16] showed that this model cannot keep the flow homogeneity in a large domain under unstable conditions and thus developed a turbulence model consistent with MOST. In Prospathopoulos et al. [13], an additional buoyancy production based on the Richardson number $R i$ was added to the turbulent kinetic energy equation to model wind turbine wakes under stable conditions. El-Askary et al. [14] further used this model and additionally applied a dissipation source term to investigate the wake behavior at different atmospheric stability conditions. These turbulence models are based on MOST and could fail in modeling wind turbine wakes when MOST breaks down under some stable conditions. As the most widely accepted way to describe the structure of the turbulent flow in the horizontally homogeneous and stationary atmospheric surface layer, MOST provides some classical similarity functions to determine the wind profile. These classical similarity functions are however only valid for the flux Richardson number $R_{f}$ below 0.25 [17]. For situations where $R_{f}>0.25$, MOST overestimates the wind shear. In general, the height of the surface boundary layer decreases with atmospheric stability down to $10 \mathrm{~m}$ in very stable cases. The decreased boundary layer height limits the wind shear and results in a lower wind shear than the one based on classical similarity functions [18].

To the best of our knowledge, there are a few simulations of the wind turbine wakes under thermally-stratified atmosphere using RANS technology in literature, which are all based on MOST. And the side effects of the breakdown of MOST on wake modeling under stable conditions have not been well investigated. The present paper aims to investigate these side effects through numerical simulations and to make a modification on MOST to eliminate these side effects. This modification is to propose a new set of similarity functions based on field measurements to limit the wind shear in very stable conditions and to introduce the new similarity functions into the turbulence model proposed by van der Laan et al. [16]. 
The remainder of this paper is organized as follows. MOST is briefly described in Section 2. Models for wind turbine wakes under stable conditions are introduced in Section 3, which cover two actuator disk model based on the thrust coefficient and based on BEM calculations, and three turbulence models for stable conditions based on MOST. Section 3 also proposes a modified turbulence model that can be consistent with arbitrary similarity functions, e.g. the new similarity functions. Breakdown of MOST under stable conditions is experimentally investigated and new similarity functions are proposed in Section 4. All the test models are studied through numerical simulations: the simulation details are described in Section 5; results of the simulations are discussed in Section 6 and then concluded in Section 7.

\section{Monin-Obukhov Similarity Theory}

According to MOST [19], any dimensionless characteristics of the turbulence depends only on the dimensionless stability parameter $\zeta=z / L$ where $z$ is the height above the surface and the Obukhov length $L$ that is defined by

$$
L \equiv-\frac{u_{*}^{3}}{\kappa \frac{g}{\Theta} \overline{w^{\prime} \theta^{\prime}}}
$$

in which $\kappa=0.4, g$ is the gravity acceleration, $\Theta$ is the time-averaged potential temperature, $\theta^{\prime}$ is the fluctuation of the potential temperature, $u_{*}=\sqrt{-\overline{u^{\prime} w^{\prime}}}$ is the friction speed where $u^{\prime}$ and $w^{\prime}$ are the fluctuations of the longitudinal and vertical velocity components.

The flow similarity functions of the gradient of velocity, the gradient of potential temperature, the turbulence kinetic energy, and its dissipation can thus be defined as:

$$
\begin{aligned}
\phi_{m}(\zeta) & \equiv \frac{\kappa z}{u_{*}} \frac{\partial U}{\partial z} \\
\phi_{h}(\zeta) & \equiv \frac{\kappa z}{\theta_{*}} \frac{\partial \Theta}{\partial z} \\
\phi_{k}(\zeta) & \equiv \frac{\sqrt{C_{\mu}}}{u_{*}^{2}} k \\
\phi_{\varepsilon}(\zeta) & \equiv \frac{\kappa z}{u_{*}^{3}} \varepsilon
\end{aligned}
$$

where $C_{\mu}=0.033, U$ is the mean streamwise speed, $k$ is the turbulence kinetic energy (TKE), $\varepsilon$ is the TKE dissipation and $\theta_{*}=-\overline{w^{\prime} \theta^{\prime}} / u_{*}$ is the scaling temperature. According to the eddy viscosity hypothesis by Boussinesq [20], we have:

$$
-\rho \overline{u^{\prime} w^{\prime}}=\mu_{t} \frac{\partial U}{\partial z}
$$

where $\rho$ is the air density and the eddy viscosity is modeled in the $k-\varepsilon$ model as [21]:

$$
\mu_{t}=\rho \frac{k^{2}}{\varepsilon}
$$

Combining the definitions of $\phi_{m}, \phi_{k}$ and $\phi_{\varepsilon}$ with Equations (6) and (7) leads to

$$
\phi_{k}(\zeta)=\sqrt{\left(\phi_{\varepsilon}(\zeta) / \phi_{m}(\zeta)\right.}
$$

Based on measurements of flows over flat terrain in the atmospheric surface layer, the classical similarity functions commonly used in literature are given as [22,23]: 


$$
\begin{gathered}
\phi_{m, \mathrm{cls}}(\zeta)= \begin{cases}\left(1-\gamma_{m} \zeta\right)^{-1 / 4} & -2<\zeta<0 \\
1+\beta_{m} \zeta & 0<\zeta<1\end{cases} \\
\phi_{h, \mathrm{cls}}(\zeta)= \begin{cases}\chi\left(1-\gamma_{h} \zeta\right)^{-1 / 2} & -2<\zeta<0 \\
\chi+\beta_{h} \zeta & 0<\zeta<1\end{cases} \\
\phi_{\varepsilon, \mathrm{cls}}(\zeta)= \begin{cases}1-\zeta & \zeta<0 \\
\phi_{m, \mathrm{cls}}-\zeta & \zeta>0\end{cases}
\end{gathered}
$$

where $\gamma_{m}=\gamma_{h}=16, \beta_{m}=\beta_{h}=5$ [23] and $\chi$ will be determined based on field measurements in Section 4 . The classical similarity functions, especially $\phi_{m}$ and $\phi_{h}$ are only valid for the flux Richardson number $R_{f}$ below 0.25 [17]. We also proposed new functions, noted as $\phi_{m, \exp }$ and $\phi_{h, \exp }$, in the full range of $R_{f}$ based on a field experiment in a wind farm.

\section{Models for Wind Turbine Wakes under Stable Conditions}

In the present work, the numerical simulation of wind turbine wakes under atmospheric stable conditions is based on RANS equations and the buoyancy effect due to atmospheric thermal stratifications is also taken into account. The following continuity, momentum, and energy equations are solved in wake simulations [15,24]:

$$
\begin{gathered}
\frac{\partial}{\partial x_{i}}\left(\rho U_{i}\right)=0 \\
\frac{\partial}{\partial t}\left(\rho U_{i}\right)+\frac{\partial}{\partial x_{j}}\left(\rho U_{i} U_{j}\right)=-\frac{\partial p}{\partial x_{i}}+\frac{\partial}{\partial x_{j}}\left[\left(\mu+\mu_{t}\right)\left(\frac{\partial U_{i}}{\partial x_{j}}+\frac{\partial U_{j}}{\partial x_{i}}\right)\right]+S_{u, i} \\
\frac{\partial}{\partial t}(\rho \Theta)+\frac{\partial}{\partial x_{i}}\left(\rho U_{i} \Theta\right)=\frac{\partial}{\partial x_{i}}\left[\left(\mu+\frac{\mu_{t}}{\sigma_{\theta}}\right) \frac{\partial \Theta}{\partial x_{i}}\right]
\end{gathered}
$$

where $\rho$ is the air density, $U_{i}$ is the velocity component in the $x_{i}$ direction, $p$ is the air pressure, $\mu$ is the laminar viscosity, $\mu_{t}$ is the turbulent viscosity, $S_{u, i}$ is the momentum term source imposed by the wind turbine rotor in the $x_{i}$ direction, $\Theta$ is the potential temperature and $\sigma_{\theta}$ is the turbulent Prandtl number.

\subsection{Modeling of Wind Turbine}

In this work, we introduce two kinds of actuator disk models: one is based on the thrust coefficient and another is based on BEM calculations to distribute forces through the disk. The second one which provides more detailed information of the distributed forces caused by the rotor, is expected to capture near wake structure better than the first one. All simulations carried out in this work use the AD model based on BEM calculations in advance if the geometry information of the blades is available.

\subsubsection{Actuator Disk Model Based on Thrust Coefficient (-CT)}

In the origin actuator disk model, the momentum source term in the $x_{i}$ direction due to the thrust is uniformly distributed through the rotor:

$$
S_{u, i}=-\frac{T}{V_{\mathrm{disk}}} \frac{U_{\mathrm{ref}, i}}{U_{\mathrm{ref}}}=-\frac{\rho C_{T} U_{\mathrm{ref}} U_{\mathrm{ref}, i}}{2 \Delta l}
$$

where $T$ is the thrust, $V_{\text {disk }}$ is the disk volume, $C_{T}$ is the thrust coefficient, $U_{\text {ref }}$ is the upstream reference velocity at hub height, $U_{\text {ref, } i}$ is the component velocity $U_{\text {ref }}$ of in $x_{i}$ direction, $\Delta l$ is the disk depth and the negative sign represents the drag effects of thrust on the flow.

For the upstream flow disturbed by a complex terrain or wind turbine wakes, $U_{\text {ref }}$ is unknown and difficult to be evaluated from the local flow directly. According to the one-dimensional momentum 
theory and ignoring the wind shear, the reference velocity is a function of the rotor-averaged velocity $U_{\text {disk: }}$

$$
U_{\text {disk }}=\left(1-a_{B}\right) U_{\text {ref }}
$$

in which the induced factor $a_{B}$ [25] is related to the thrust coefficient $C_{T}$ by

$$
a_{B}= \begin{cases}\frac{1}{2}\left(1-\sqrt{1-C_{T}}\right), & C_{T} \leq \frac{8}{9} \\ \frac{C_{T}-4 a_{c}^{2}}{4\left(1-2 a_{c}\right)^{\prime}}, & C_{T}>\frac{8}{9}\end{cases}
$$

where $a_{c}=1 / 3$.

In simulations, the disk-averaged velocity $U_{\text {disk }}$ is calculated by averaging the local velocity in the disk region, then is applied to estimate the upstream reference velocity $U_{\text {ref }}$ based on Equations (16) and (17) and the momentum source $S_{u, i}$ based on Equation (15), respectively.

\subsubsection{Actuator Disk Model Based on Blade Element Method (-BEM)}

In the BEM-based actuator disk model, the rotor plane consists of $N$ actuator lines and each of the actuator lines is split into $M$ element sections (Fig. 1). The element section collects the local velocity and the rotor speed $\Omega$ to calculate the element force and applies this force in the neighbor cells of the element section. The reference velocity is firstly assessed from the disk-averaged velocity and then is applied to evaluate the rotor speed.

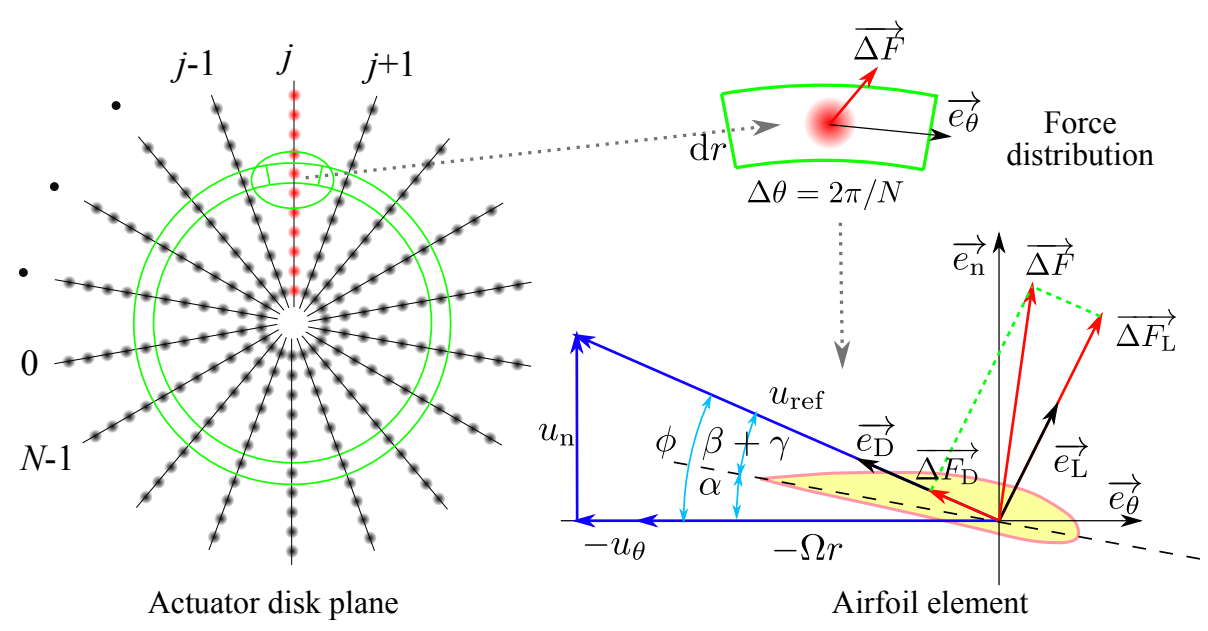

Figure 1. Schemtaics of the BEM-based actuator disk model.

By transforming the local velocity at the blade element into polar velocity components $\left(u_{r}, u_{\theta}\right.$, $u_{n}$ ), the force of the blade element is:

$$
\overrightarrow{\Delta F}=\frac{B \Delta \theta}{4 \pi} \rho u_{r e l}^{2} c\left(C_{L} \overrightarrow{e_{L}}+C_{D} \overrightarrow{e_{D}}\right) \Delta r
$$

where $B$ is the number of the blades and $c$ is the chord length, $\Delta r$ is the length of the element section. The drag coefficient of the element section, $C_{D}$ and its lift coefficient $C_{L}$ which are functions of the attack angle $\alpha$, are estimated from XFOIL [26] and then corrected by three-dimensional rotational effects of the blades based on Du et al. [27]. According to Fig. $1, \alpha=\phi-(\beta+\gamma)$ where $\phi=\arctan \left[u_{\mathrm{n}} /\left(\Omega r+u_{\theta}\right)\right]$ is the flow angle, $\beta$ is the blade installation angle and $\gamma$ is the pitch angle.

The element force is distributed across neighbor cells. The force added to a cell is calculated by:

$$
\overrightarrow{\Delta F_{\text {cell }}}=\sum_{i}^{N \cdot M} \frac{1}{s^{3} \pi^{3 / 2}} \exp \left(-\frac{s_{i}^{2}}{s^{2}}\right) \overrightarrow{\Delta F}_{i} F_{\text {tip }} F_{\text {hub }} \Delta V_{\text {cell }}
$$


where $s_{i}$ the distance of the $i$-th element to the cell and $s$ is the cut-off length scale that takes a value between 2 and 3 cell sizes [28]. $F_{\text {tip }}$ and $F_{\text {hub }}$ are the Prandtl tip loss and hub loss functions [29]:

$$
\begin{gathered}
F_{\text {tip }}=\frac{2}{\pi} \arccos \left[\exp \left(\frac{B(R-r)}{2 r \sin \phi}\right)\right] \\
F_{\text {hub }}=\frac{2}{\pi} \arccos \left[\exp \left(\frac{B\left(r-R_{\text {hub }}\right)}{2 r \sin \phi}\right)\right]
\end{gathered}
$$

where $R$ is the rotor radius, $R_{\text {hub }}$ is the hub radius, $r$ is the radial distance of the element to the rotor center.

\subsection{Turbulence modeling}

In this paper, we apply the $k-\varepsilon$ turbulence model to close Equations (12) to (14):

$$
\begin{gathered}
\frac{\partial}{\partial t}(\rho k)+\frac{\partial}{\partial x_{i}}\left(\rho U_{i} k\right)=\frac{\partial}{\partial x_{j}}\left[\left(\mu+\frac{\mu_{t}}{\sigma_{k}}\right) \frac{\partial k}{\partial x_{j}}\right]+\mathcal{P}+\mathcal{B}-\rho \varepsilon-S_{k, \mathrm{SBL}} \\
\frac{\partial}{\partial t}(\rho \varepsilon)+\frac{\partial}{\partial x_{i}}\left(\rho U_{i} \varepsilon\right)=\frac{\partial}{\partial x_{j}}\left[\left(\mu+\frac{\mu_{t}}{\sigma_{\varepsilon}}\right) \frac{\partial \varepsilon}{\partial x_{j}}\right]+\left(C_{\varepsilon 1} \mathcal{P}-\rho C_{\varepsilon 2} \varepsilon+C_{\varepsilon 3} \mathcal{B}\right) \frac{\varepsilon}{k}+S_{\varepsilon, \text { wake }} \\
\mu_{t}=\rho C_{\mu} \frac{k^{2}}{\varepsilon}
\end{gathered}
$$

where $k$ is the turbulence kinetic energy (TKE), $\varepsilon$ is the TKE dissipation, $C_{\mu}=0.033, \sigma_{k}=1.0, \sigma_{\varepsilon}=$ $1.3, C_{\varepsilon 2}=1.92$ and $C_{\varepsilon 3}$ is a coefficient to be calibrated with atmospheric stability. $\mathcal{P}$ and $\mathcal{B}$ are the TKE source production due to shear and buoyancy:

$$
\begin{gathered}
\mathcal{P} \equiv-\rho \overline{u_{i}^{\prime} u_{j}^{\prime}} \frac{\partial U_{j}}{\partial x_{i}}=\mu_{t}\left(\frac{\partial U_{i}}{\partial x_{j}}+\frac{\partial U_{j}}{\partial x_{i}}\right) \frac{\partial U_{j}}{\partial x_{i}} \\
\mathcal{B} \equiv \frac{g_{i}}{\Theta_{0}} \overline{u_{i}^{\prime} \theta^{\prime}}=-\frac{g_{i}}{\Theta_{0}} \frac{\mu_{t}}{\sigma_{\theta}} \frac{\partial \Theta}{\partial x_{i}}
\end{gathered}
$$

where $u_{i}^{\prime}$ is the fluctuation of the velocity component in $x_{i}$ direction.

The source term $S_{\varepsilon, \text { wake }}$ is applied in the neighbour region of the turbine to correct the fast wake recovery of the standard $k-\varepsilon$ model [30]:

$$
S_{\varepsilon, \text { wake }}=\rho C_{4 \varepsilon} \frac{\mathcal{P}^{2}}{\rho k}
$$

where $C_{4 \varepsilon}=0.37$.

In this work the methods of Alinot and Masson [15], the recently proposed model from M.P. van der Laan et al. [16] and the indirect model that not solves the energy equation applied in W.A. El-Askary et al. [14] are investigated, and the models are hereafter referred to as the AM, Laan and El-Askary models, respectively. The TKE source term $S_{\varepsilon, \mathrm{ABL}}$ is added in the Laan model and its modified model (the proposed model) to keep flow homogeneity under stable conditions. In the AM model and the El-Askary model, $S_{k, \mathrm{ABL}}=0$. For stable cases, $C_{\varepsilon 3}$ is set to -2.9 in the AM model [15] and set to 1 in the El-Askary model [14].

In the El-Askary model, the energy equation (14) is not solved and the TKE production due to buoyancy $\mathcal{B}$ is then modeled as:

$$
\mathcal{B}=-\mu_{t}\left(\frac{\partial u}{\partial z}\right)^{2} \frac{R i}{\phi_{m}}
$$

in which 


$$
R i=\zeta \frac{0.74+4.7 \zeta}{(1+4.7 \zeta)^{2}}, \quad \zeta>0
$$

The Laan model is derived from homogeneous steady flows over flat terrain where the kinematic viscosity $\mu$ can be ignored and the $k-\varepsilon$ model can be rewritten into normalized form by $\kappa z / u_{*}^{3}$ :

$$
\begin{gathered}
\phi_{t, k}+\phi_{m}-\phi_{\varepsilon}-\frac{\phi_{h}}{\sigma_{\theta} \phi_{m}} \zeta-\phi_{S_{k}}=0 \\
\phi_{t, \varepsilon}+\left(C_{\varepsilon 1} \phi_{m}-C_{\varepsilon 2} \phi_{\varepsilon}-C_{\varepsilon 3} \frac{\phi_{h}}{\sigma_{\theta} \phi_{m}} \zeta\right) \frac{\varepsilon}{k}=0
\end{gathered}
$$

in which $\phi_{t, k} \equiv \frac{\kappa z}{\rho u_{*}^{3}} \frac{\partial}{\partial z}\left(\frac{\mu_{t}}{\sigma_{k}} \frac{\partial k}{\partial z}\right)$ and $\phi_{t, \varepsilon} \equiv \frac{\kappa z}{\rho u_{*}^{3}} \frac{\partial}{\partial z}\left(\frac{\mu_{t}}{\sigma_{\varepsilon}} \frac{\partial \varepsilon}{\partial z}\right)$ are the normalized turbulent transport of $k$ and $\varepsilon, \phi S_{k} \equiv \frac{\kappa z}{u_{*}^{3}} S_{k, \mathrm{ABL}}$.

Under neutral conditions where $\zeta \rightarrow 0$ and $\phi_{m}, \phi_{k}, \phi_{\varepsilon} \rightarrow 1$, Eq. (31) reduces to

$$
C_{\mu}^{1 / 2} \sigma_{\varepsilon}\left(C_{\varepsilon 2}-C_{\varepsilon 1}\right)=\kappa^{2}
$$

which results in $C_{\varepsilon 1}=1.24$.

Under stable conditions, we have

$$
\begin{gathered}
S_{k, \mathrm{ABL}}=\left\{\phi_{m}-\phi_{\varepsilon}-\frac{\phi_{h}}{\sigma_{\theta} \phi_{m}} \zeta+\frac{C_{\mu}^{-1 / 2} \kappa^{2}}{\sigma_{k}} \frac{\zeta^{2}}{\phi_{m}}\left[\phi_{k}^{\prime \prime}-\frac{\phi_{k}^{\prime} \phi_{m}^{\prime}}{\phi_{m}}+\frac{\phi_{k}^{\prime}}{\zeta}\right]\right\} \frac{u_{*}^{3}}{\kappa z} \\
\frac{\phi_{h}}{\sigma_{\theta} \phi_{m}} C_{\varepsilon 3}=\frac{C_{\varepsilon 1} \phi_{m}-C_{\varepsilon 2} \phi_{\varepsilon}}{\zeta}+\frac{C_{\mu}^{-1 / 2} \kappa^{2}}{\sigma_{\varepsilon}} \frac{\phi_{k}}{\phi_{m}}\left[\frac{\zeta \phi_{\varepsilon}^{\prime \prime}}{\phi_{\varepsilon}}-\frac{\zeta \phi_{\varepsilon}^{\prime} \phi_{m}^{\prime}}{\phi_{\varepsilon} \phi_{m}}-\frac{\phi_{\varepsilon}^{\prime}}{\phi_{\varepsilon}}+\frac{\phi_{m}^{\prime}}{\phi_{m}}+\frac{1}{\zeta}\right]
\end{gathered}
$$

where $\phi^{\prime}=\partial \phi / \partial \zeta, \phi^{\prime \prime}=\partial^{2} \phi / \partial \zeta^{2}$ for $\phi \in\left\{\phi_{k}, \phi_{m}, \phi_{\varepsilon}\right\}$. Equations (33) and (34) allow turbulence closure consistent either with the classical similarity functions in MOST (the Laan model) or any other similarity functions based on field measurements (the proposed model in this paper). One can prove that the above equations equal to those proposed by van der Laan et al. [16] if the classical similarity functions are applied. It should be noted that the turbulent Prandtl number is set to 1 in the origin AM model and the Laan model, and the energy equation (14) is also not solved in the Laan model. This work however apply $\sigma_{\theta} \equiv \phi_{h} / \phi_{m}$ according to the definition of the turbulent Prandtl number [31] and solves the energy equation (14) in the Laan model. There are only negligible differences in wake modeling when considering these differences in implementations of the models. Table 1 summarizes the equations to be solved as well as the applied source terms, used for each method.

Table 1. Summary of turbulence models investigated for stable conditions.

\begin{tabular}{cccccc}
\hline Turbulence Model & Energy Equation (14) & $\mathcal{B}$ & $S_{k, \mathrm{ABL}}$ & $C_{3 \varepsilon}$ & Similarity functions \\
\hline AM & $\sqrt{ }$ & Eq. (26) & - & -2.9 & classical \\
El-Askary & - & Eq. (28) & - & 1 & classical \\
Laan & $\sqrt{ }$ & Eq. (26) & Eq. (33) & Eq. (34) & classical \\
Proposed & $\sqrt{ }$ & Eq. (26) & Eq. (33) & Eq. (34) & $\phi_{m, \exp , \phi_{h, \exp }, \phi_{\varepsilon, \mathrm{cls}}}$ \\
\hline
\end{tabular}

\section{Breakdown and Modifications of MOST under Stable Conditions}

\subsection{Experimental Data}

The field experiment that is used to investigate the similarity functions was carried out in a wind farm in the Jingbian wind farm in northwest of China [2]. In the experimental campaign, two masts numbered M1 and M3 are installed near a wind turbine (No. 14) to capture the wake profiles (Fig. 2). For wind direction in $\left[-17^{\circ}, 8^{\circ}\right]$, the ratio of the nacelle wind speed of wind turbine 14 to the 
hub-height wind speed at mast M1 keeps constant which indicates mast M1 is not located in wakes of turbines NO. 12 and No. 15, we thus use the data of mast M1 in this wind direction interval to study similarity functions. On the mast M1, cup anemometers were installed at $30 \mathrm{~m}, 50 \mathrm{~m}$ and $70 \mathrm{~m}$ (Fig. 3). Measurements of the sonic anemometer installed on $\mathrm{M} 1$ at $30 \mathrm{~m}$ are applied to estimate the heat flux $\overline{w^{\prime} \theta^{\prime}}$, the Obukhov length $L$ and the friction speed $u_{*}$ [2]. Effective data after considering data synchronization and sensor errors are about 190 days.

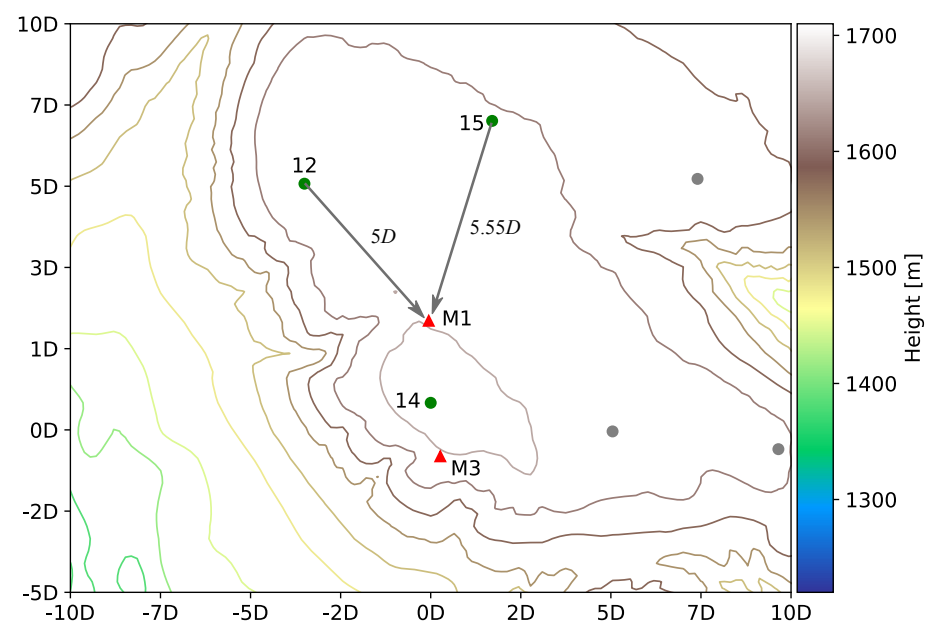

Figure 2. Complex terrain around M1.

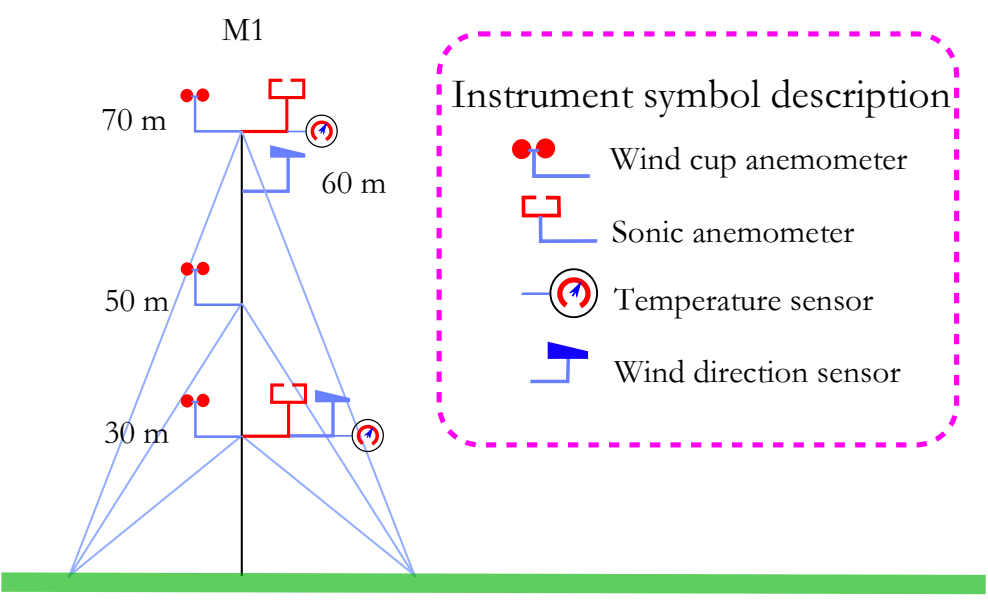

Figure 3. Measurement setup for M1.

According to Eq. (2), the vertical gradient of velocity is also required to estimate $\phi_{m}$ besides the friction speed $u_{*}$. In this work, we approximate the vertical gradient of velocity by:

$$
\left.\frac{d U}{d z}\right|_{30 \mathrm{~m}}=\frac{-3 U_{30}+4 U_{50}-U_{70}}{40 \mathrm{~m}}
$$

where $U_{30}, U_{50}$ and $U_{70}$ is the speed at $30 \mathrm{~m}, 50 \mathrm{~m}$ and $70 \mathrm{~m}$ above the ground level (a.g.l.). Due to the absence of a temperature sensor at $50 \mathrm{~m}$ a.g.l., we can only estimate $\phi_{h}(\zeta)$ with second-order accuracy at $50 \mathrm{~m}$. In order to estimate $\phi_{h}$ at $30 \mathrm{~m}$ with second-order accuracy, the turbulent Prandtl number $\sigma_{\theta}=\phi_{h} / \phi_{m}$ is considered to be independent of heights. Then the turbulent Prandtl number assessed at $50 \mathrm{~m}$ a.g.1. is also available at $30 \mathrm{~m}$ a.g.l. The vertical gradient of speed and potential temperature in estimating $\sigma_{\theta}$ at $50 \mathrm{~m}$ are given by Wallace et al. [32]: 


$$
\begin{gathered}
\left.\frac{d U}{d z}\right|_{50 \mathrm{~m}}=\frac{U_{70}-U_{30}}{40 \mathrm{~m}} \\
\left.\frac{d \Theta}{d z}\right|_{50 \mathrm{~m}}=\frac{T_{70}-T_{30}}{40 \mathrm{~m}}+g / C_{p}
\end{gathered}
$$

\subsection{Limitations and Breakdown of MOST}

The flux Richardson number is defined as:

$$
R_{f} \equiv-\frac{\frac{g}{\Theta} \overline{w^{\prime} \theta^{\prime}}}{u_{*}^{2} \frac{\partial U}{\partial z}}
$$

Combining the definitions of $L$ and $R_{f}$, we have

$$
\phi_{m}(\zeta)=\zeta / R_{f}
$$

which indicates that $R_{f}=0.25$ is a straight line in the plane $\zeta-\phi_{m}$. Fig. 4 shows that the line $R_{f}=0.25$ split the measured data under stable conditions into two groups: for $R_{f}<0.25$, the measured data have good agreements with the data predicted by MOST and are rarely observed above $\zeta=2$; for $R_{f}>0.25$, the measured data have smaller $\phi_{m}$ than those predicted by MOST. For applications in wind energy, measured data are usually mixed and engineers commonly focus on wind turbine wakes under stable conditions without considering the limitation of $R_{f}$. In this situation, MOST could overestimate the wind shear and potentially influence wake modeling of a wind turbine.

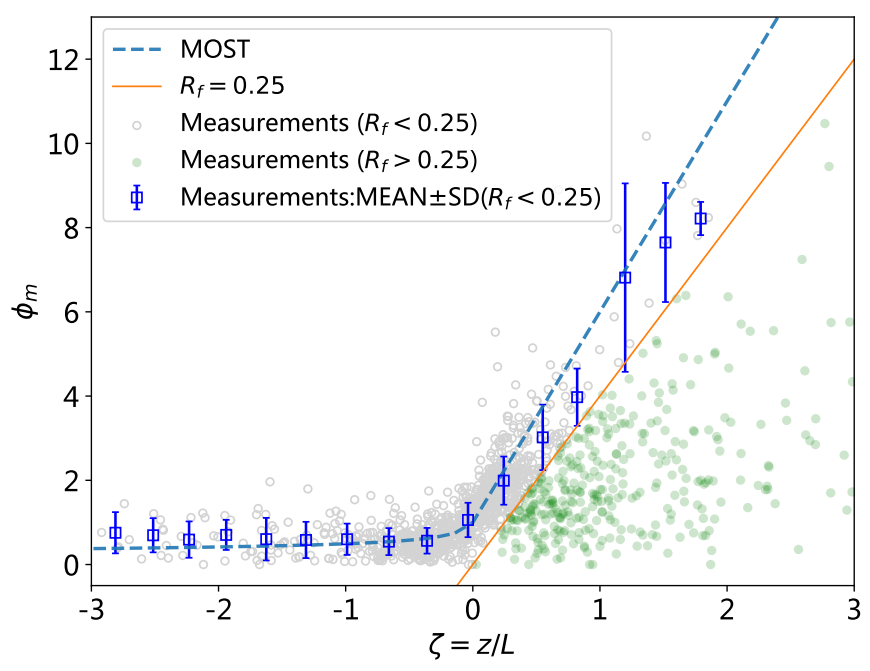

Figure 4. Plot of $\phi_{m}$ as function of $\zeta$.

\subsection{The Proposed Similarity Functions}

Fig. 5 and Fig. 6 show comparisons of $\phi_{m}(\zeta)$ and $\sigma_{\theta}(\zeta)$ of different models with classical similarity functions of MOST. Measurements show that $\phi_{m}$ of MOST only be valid for $\zeta<0.1$ and is overestimated for $\zeta>0.1$. Fig. 6 show that the turbulent Prandtl number is decreased with the stability parameter $\zeta$. A similar phenomenon was also observed in Grachev et al. [31]. In the full range of $R_{f}$, the proposed similarity functions based on a field measurement under stable conditions read:

$$
\phi_{m, \exp }(\zeta)=(1+6 \zeta)^{\frac{1+16 \zeta^{2}}{1+40 \zeta^{2}}}
$$




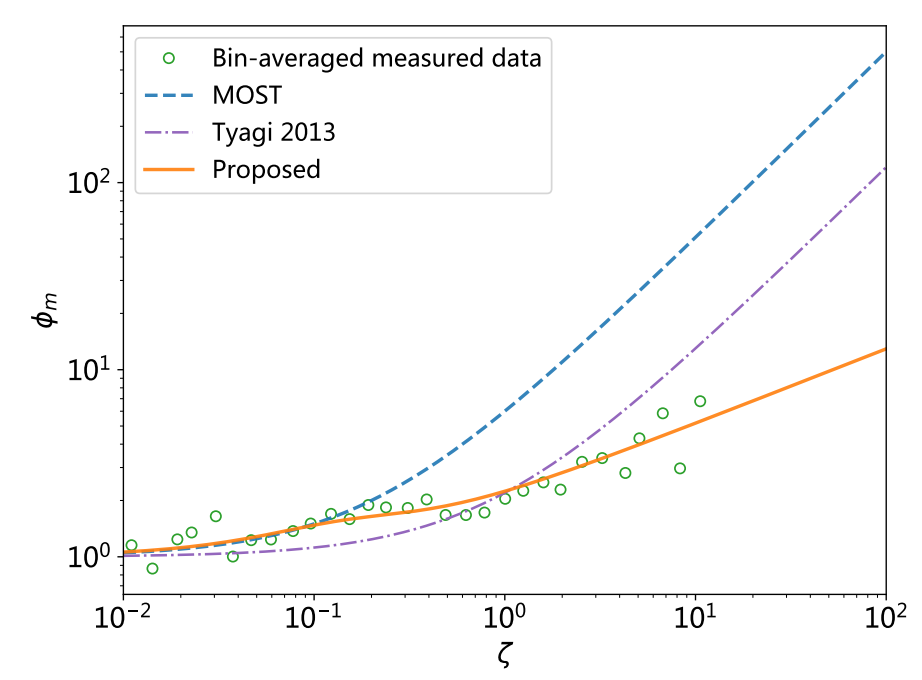

Figure 5. Plots of $\phi_{m}$ of different models as functions of $\zeta$.

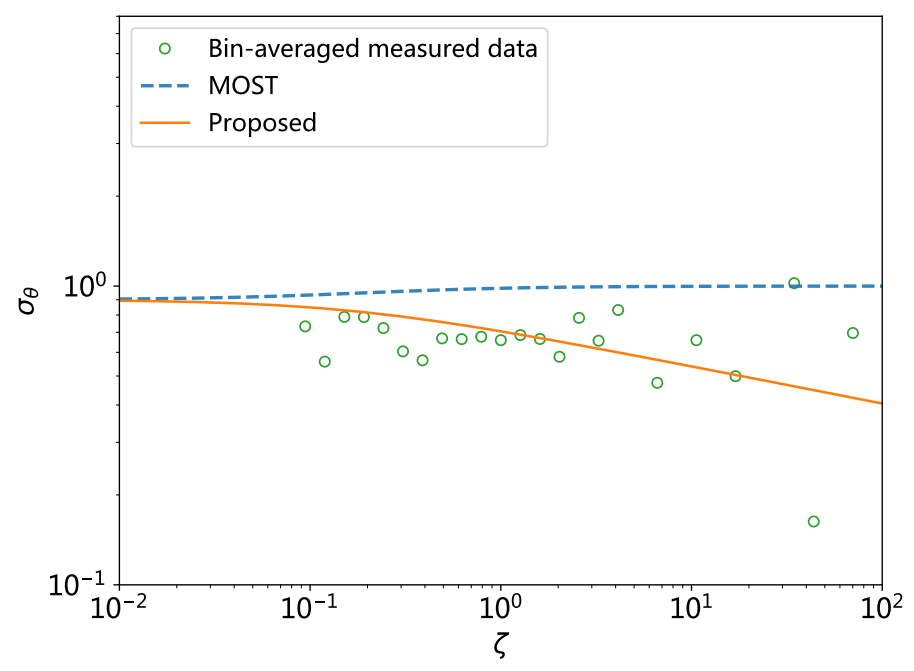

Figure 6. Plots of $\sigma_{\theta}$ of different models as functions of $\zeta$.

$$
\sigma_{\theta, \exp }(\zeta)=0.9(1+6 \zeta)^{-1 / 8}
$$

224 where $\sigma_{\theta, \exp }(\zeta)=\phi_{h, \exp }(\zeta) / \phi_{m, \exp }(\zeta)$ and $\chi=0.9$. To note, Eq. (40) approximates $1+6 \zeta$ for $\zeta<0.1$ and approximates $(1+6 \zeta)^{0.25}$ for $\zeta \rightarrow \infty$, which allows to limit wind shear under strongly stable conditions.

\section{Simulation Details}

\subsection{Test Cases}

In this work, we investigate wakes under stable conditions of a $500 \mathrm{~kW}$ NTK500/41 wind turbine at the Risø Campus test site of DTU in Denmark [1] and three $180 \mathrm{~kW}$ Danwin turbines on the island of Gotland in the Baltic Sea [6]. The two test cases are based on wake measurements that cover wake data $1 D$ to $9 D$ downstream of the turbines where $D$ is the rotor diameter. Details of test cases are listed in Tab. 2. 
Table 2. Details of measurements in the two test cases.

\begin{tabular}{ccccc}
\hline Wind turbine & $D(\mathbf{m})$ & $H(\mathbf{m})$ & Measurements & Wake range \\
\hline NTK500/41 & 41 & 36 & LiDAR scanning & $1 D$ to $5 D$ \\
Danwin & 23 & 35 & Mast measurements & $4.2 D, 6.1 D$ and $9.6 D$ \\
\hline
\end{tabular}

The first test case is based on LiDAR measurements of the wakes of a NTK500/41 turbine. A pulsed LiDAR which was mounted on a platform at the rear of the nacelle, pointed its laser downstream the turbine up to $5 \mathrm{D}$ downstream the turbine. A constant downhill slope of about $0.3 \%$ was observed downstream the turbine. Inlet meteorological properties such as wind speed, wind direction, air temperature and atmospheric pressure were measured from a $57 \mathrm{~m}$ tall meteorological mast located upstream the turbine. The NTK500/41 turbine is a stall-regulated $500 \mathrm{~kW}$ wind turbine equipped with LM $19.1 \mathrm{~m}$ blades and its rotor speed is fixed at $27.1 \mathrm{rpm}$. The blades consist of two kinds of airfoils: FFA-W3-XX1 (from 16\% to 50\% span) and NACA 63-XXX (from 60\% to 100\% span) [33]. Chord length and twist angle over the blades were presented in Johansen et al. [34]. The power curve and the thrust coefficient curve based on BEM calculations are shown in Fig. 7. The power curve from BEM calculations is shown to have good agreements with the RANS computations in fully turbulence model [34] below $15 \mathrm{~m} / \mathrm{s}$ and measurements [34] below $10 \mathrm{~m} / \mathrm{s}$ (Fig. 7). The measured thrust coefficient from strain gauges measurements [35] is shown to have good agreements with the BEM computations above $6 \mathrm{~m} / \mathrm{s}$. In this case, we set $z_{0}=0.095 \mathrm{~m}, L=29 \mathrm{~m}$, the hub-height reference wind speed $U_{\text {hub }}=6.76$ $\mathrm{m} / \mathrm{s}$ as presented in Machefaux et al. [1] and apply $C_{T}=0.83$ based on the BEM computations.

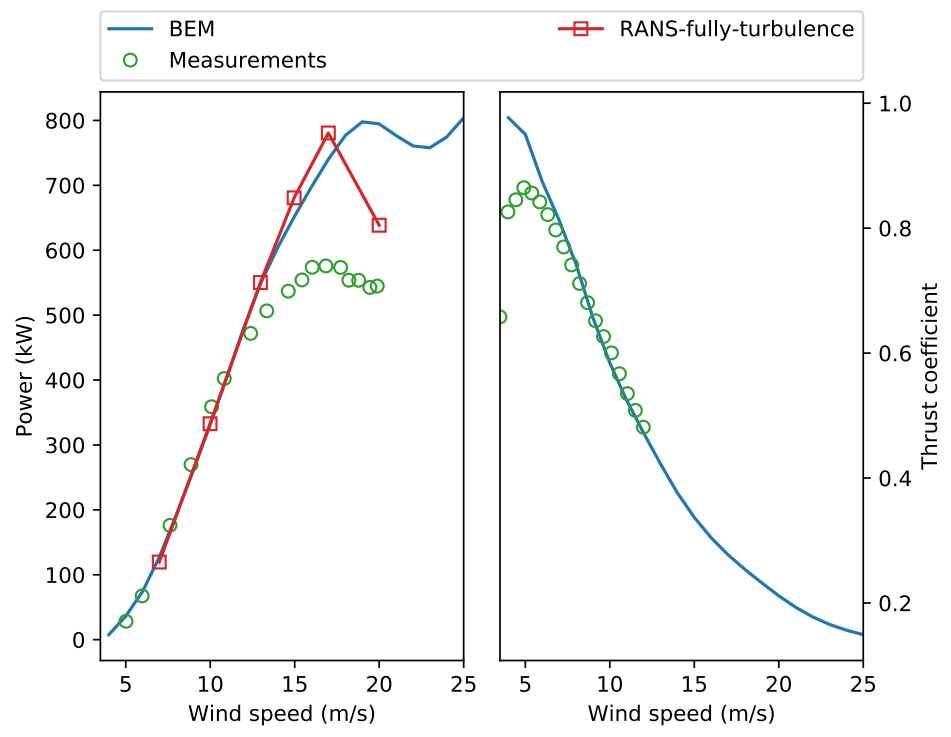

Figure 7. Performance curves of the NTK500/41 turbine.

The second test case is based on wake measurements of three Danwin wind turbines using two 54 m meteorological masts, M1 and M2. In the campaign, the masts were equipped with wind sensors at 8 levels from $10 \mathrm{~m}$ to $53.3 \mathrm{~m}$. Inlet conditions including temperature profiles were measured on mast M1 while wake profiles of the three turbines were measured on mast M2. The distances from the three turbines to mast M2 are $4.2 D, 6.1 D$ and $9.6 D$, respectively. In this case, we set the roughness length, the Obukhov length, the reference wind speed and the thrust coefficient to be $0.0005 \mathrm{~m}, 35 \mathrm{~m}, 8$ $\mathrm{m} / \mathrm{s}$ and 0.82 , respectively [6,36]. The detail information of test cases is shown in Tab. 3 where the model "Proposed-z0" is actually the proposed model that reduces $z_{0}$ to impose the turbulent intensity at hub height equivalent to the value imposed by MOST. The model "Proposed-z0" is used to assess the influence of turbulence intensity on wake modeling. 
Table 3. Test cases and corresponding parameters under stable conditions with $L=29 \mathrm{~m}$ for Case 1 and $L=35 \mathrm{~m}$ for Case 2 where TI stands for turbulence intensity at hub height.

\begin{tabular}{ccccccc}
\hline \multirow{2}{*}{ Model } & \multicolumn{2}{c}{ Case 1: $U_{\text {hub }}=6.76 \mathrm{~m} / \mathrm{s}, C_{T}=0.83$} & \multicolumn{3}{c}{ Case 2: $U_{\text {hub }}=8 \mathrm{~m} / \mathrm{s}, C_{T}=0.82$} \\
\cline { 2 - 7 } & $u_{*}(\mathrm{~m} / \mathrm{s})$ & $z_{0}(\mathrm{~m})$ & TI & $u_{*}(\mathrm{~m} / \mathrm{s})$ & $z_{0}(\mathrm{~m})$ & TI \\
\hline AM, Laan, El-Askary & 0.223 & 0.095 & $6.3 \%$ & 0.198 & 0.0005 & $4.7 \%$ \\
Proposed & 0.316 & 0.095 & $9 \%$ & 0.237 & 0.0005 & $5.7 \%$ \\
Proposed-z0 & 0.223 & 0.0028 & $6.3 \%$ & - & - & - \\
\hline
\end{tabular}

\subsection{Computational Domain and Meshing}

The computational domain has a length of $20 D$, a width of $10 D$ and a height of $10 D$ (Fig. 8). The background mesh whose refinement level is 0 , consists of $100 \times 60 \times 60$ in length, width and height. The vertical grids are clustered near the ground and the first cell height above the ground is set to 7.38 $z_{0}$ to produce appropriate kinetic energy [37]. The mesh around the wind turbine and in the wake region is refined as shown in Fig. 8. The mesh is refined around the disk region to ensure about 80 cells through the rotor diameter [38]. The complete mesh is comprised of about 1.6 million cells.
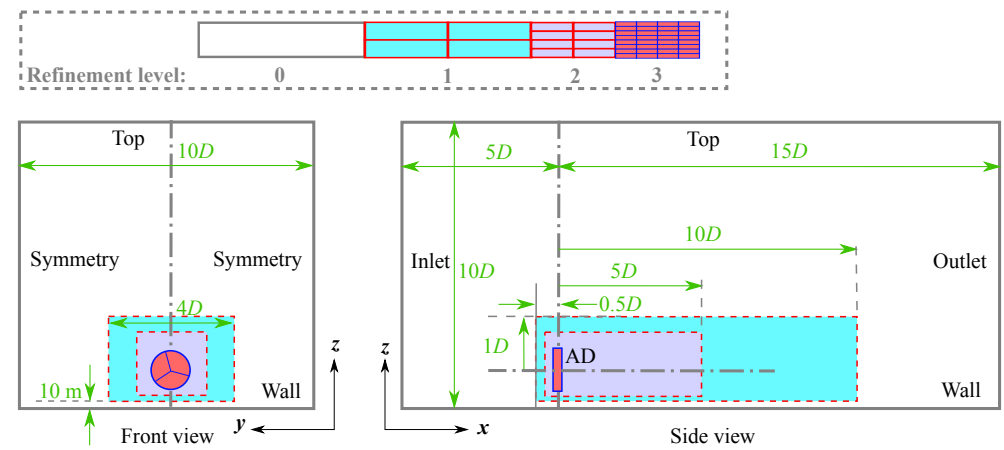

Figure 8. Compuational domain and meshing settings.

\subsection{Boundary Conditions and Solver Settings}

The boundary conditions consistent with similarity functions are applied to modeling the atmospheric boundary stratification. We apply the following inlet conditions in wake simulations:

$$
\begin{gathered}
U(z)=\int_{z_{0}}^{z} \frac{u_{*}}{\kappa z} \phi_{m}\left(\frac{z}{L}\right) \mathrm{d} z \\
\Theta(z)=\Theta_{0}+\int_{z_{0}}^{z} \frac{\theta_{*}}{\kappa z} \phi_{h}\left(\frac{z}{L}\right) \mathrm{d} z \\
\varepsilon=\frac{u_{*}^{3}}{\kappa z} \phi_{\varepsilon}\left(\frac{z}{L}\right) \\
k=\frac{u_{*}^{2}}{\sqrt{C_{\mu}}} \phi_{k}\left(\frac{z}{L}\right)
\end{gathered}
$$

The vertical profiles of wind speed and potential temperature are estimated in numerical integration. Zero gradients of $U, \Theta, \varepsilon, k$ are applied at the outlet. For the top boundary, the upstream flow properties are maintained constant. And the turbulent law of wall presented in Chang et al. [39] is applied to the first layer of cells above the wall. The left and right sides of the computational domain are set to be symmetry.

All the AD models are implemented in OpenFOAM (Open-source Field Operation And Manipulation) [40], which is a C++ toolbox for the development of customized numerical 
solvers, and pre-/post-processing utilities for allowing for solutions to fluid flow problems using the finite-volume method. To carry out the simulations, we develop a new solver based on buoyantBoussinesqPimpleFoam which is a large time-step transient solver using the PIMPLE (merged PISO-SIMPLE) algorithm for buoyant, turbulent flow of incompressible fluids provided in OpenFOAM. A first-order upwind scheme is used for all dependent variables in all the simulations. Momentum source $S_{u}$ is added to the momentum equations via user-specified finite volume options (fvOptions).

\section{Results of Wake Simulations}

\subsection{Case 1: Wakes of a NTK500/41 Wind Turbine}

In Machefaux et al. [1], the wakes of a NTK500/41 wind turbine was studied experimentally and numerically by using two LES methods: the classical one based on the ELLIPSYS3D flow solver [41] and the extended approach that explicitly introduces thermal and Coriolis effects as external force terms into the solver. The modeled Monin-Obukhov inlet was observed to be much more severely sheared as compared with measurement such that the classical approach applied a power law for the inlet velocity profile. The extended LES approach additionally carried out a transient precursor computation to simulates the time-varying vertical structure of the whole ABL and used the results of the precursor simulations to impose the mean potential temperature and velocity profiles at the inlet for wake modeling. The precursor simulations which are shown to have effects on limiting the overestimated wind shear predicted by MOST [1], are not carried out for the various turbulence models combined with RANS in this work. Besides, numerical simulations have shown that there are no major differences in wake deficit predictions between the classical approach and the extended model for stable and unstable cases. Therefore, we only compare the wake deficit predicted by the extended approach with the test models in RANS in this work.

Fig. 9 shows contours of wake deficits $\Delta U / U_{0}$ predicted by different models at hub height under stable conditions where $\Delta U$ is the velocity deficit in the wake and $U_{0}$ is the inlet velocity. The simulations using LES technology show larger deficits in near wake and a slightly faster recovery wake than that in the proposed model, while the Laan model predicts a slower recovery wake with a much larger deficit. One possible explanation for the stronger wake effects predicted by the Laan model is due to the underpredicted turbulence intensity. For a given reference wind speed $U_{\text {hub }}$ and a fixed roughness length $z_{0}$, higher wind shear in the Laan model results in a smaller friction speed $u_{*}$ and a lower turbulence intensity $I_{\text {hub }}$ than the proposed model and thus weakens the wake recovery. In this case, the ambient turbulence intensity $9 \%$ in the LES approach and the proposed model, is reduced to $6.3 \%$ in the Laan model. Fig. 9(c) shows the effects of the reduced turbulence intensity on wake modeling. Compared to the significant difference in the wake prediction between the proposed model and the Laan model, reducing the turbulence intensity slightly increases the wake deficit and weakens the wake recovery. This suggests that the overestimated wake effects by MOST are related to both the underpredicted turbulence intensity and the overestimated wind shear. 


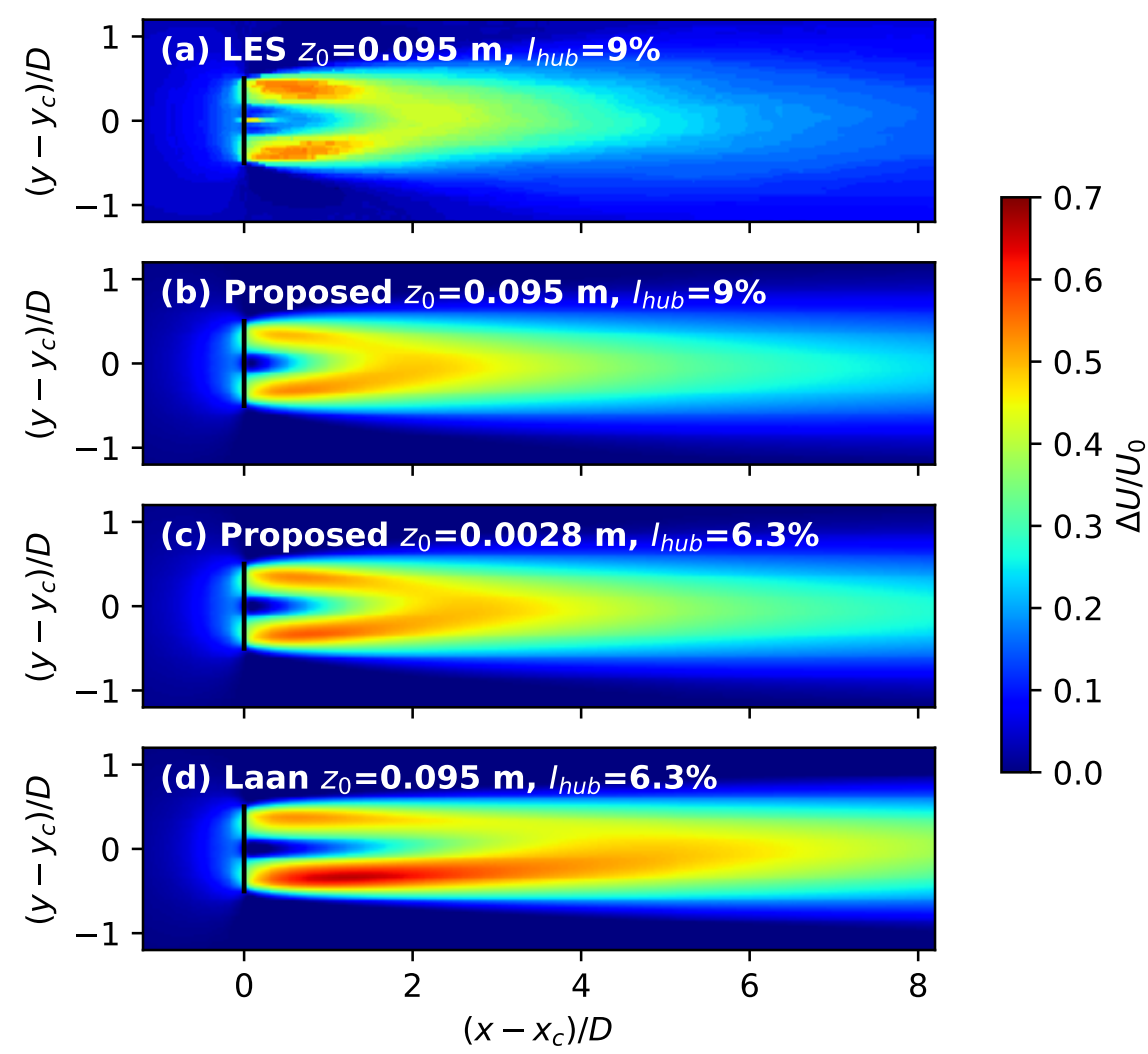

Figure 9. Normalized velocity distribution at hub height in the stable case where $\left(x_{c}, y_{c}, z_{c}\right)$ is the center point of the disk, $\Delta U$ is the velocity deficit in the wake and $U_{0}$ is the inlet velocity.

As compared with measurements, the proposed model shows better performance than other approaches based on MOST combined with RANS and is comparable to the LES approach (Fig. 10-11). For the longitudinal distance above $2 \mathrm{D}, \mathrm{AM}$, El-Askary and Laan model overestimate the wake deficits in both vertical and lateral directions. All the test models in RANS fail to predict wake deficits at $5 D$ downstream of the rotor. This disagreement is probably due to a combination of terrain effects and experimental uncertainties using LiDAR. According to the Space Shuttle Topography Mission (STRM)-based terrain data, the test site terrain has a downhill slope characterized by a height difference of $5.5 \mathrm{~m}$ from the rotor location to the most downstream cross section. As the wake has been observed to follow terrain under stable conditions [1,42], the wind speed for the longitudinal distance above $5 \mathrm{D}$ at the hub height is actually the value of $5.5 \mathrm{~m}$ above the wake center, which results in the overestimation of wake by various turbulence models in RANS. 


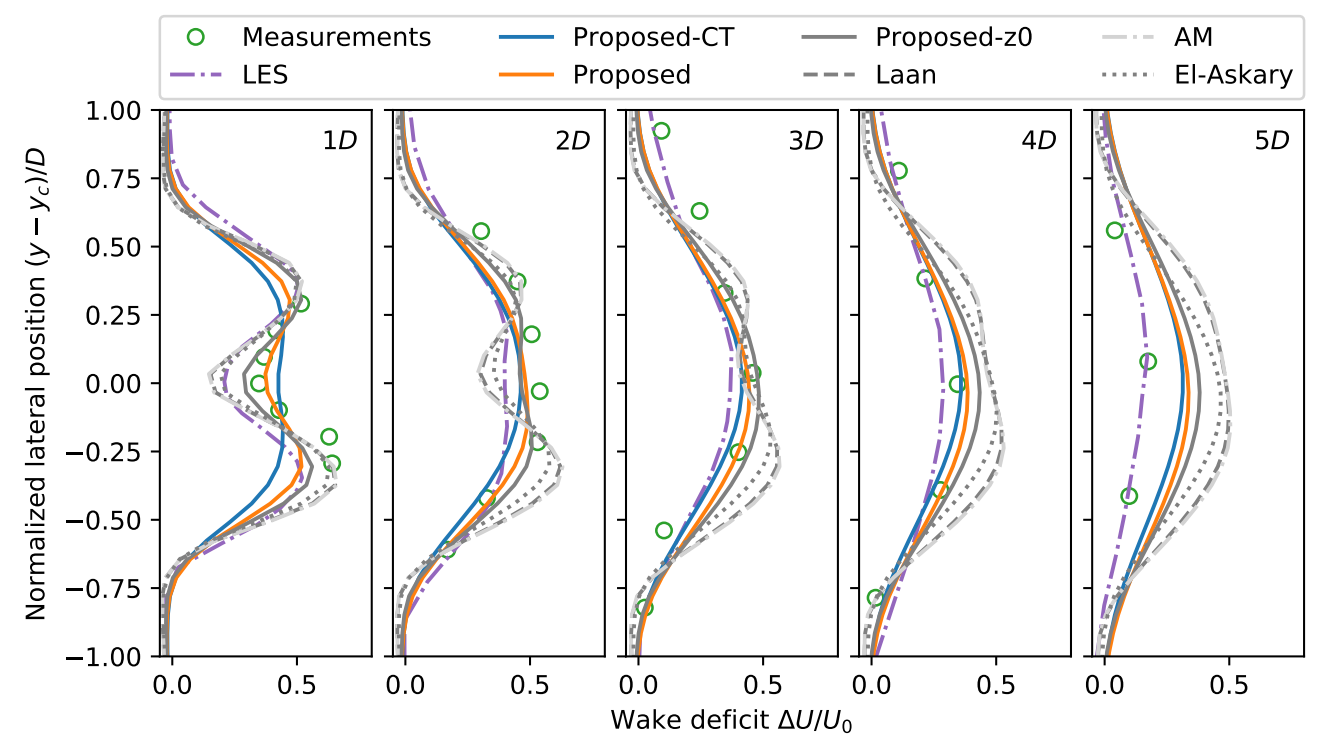

Figure 10. Lateral wake deficit of a NTK500/41 wind turbine at hub height: $L=29 \mathrm{~m}, \Delta U$ is the velocity deficit in the wake and $U_{0}$ is the inlet velocity.

Fig. 11 also shows that the proposed model underestimates wake deficits above the hub height at $1 D$ and $2 D$ while overestimating wake deficits below the hub height at $5 D$. Double-bell near-wake shape due to a lower energy extraction around the blade root [1], is observed under stable coditions at $1 D$ and is captured by the actuator models based on BEM calculations. There are no major differences between the vertical wake profiles predicted by the proposed models based on either the thrust coefficient (Proposed-CT) or BEM calculations (Proposed) above 2D. As the LES model applies slip wall conditions at the bottom of the domain and does not consider the roughness effect of the ground, large negative wake deficits are observed near the ground [1].

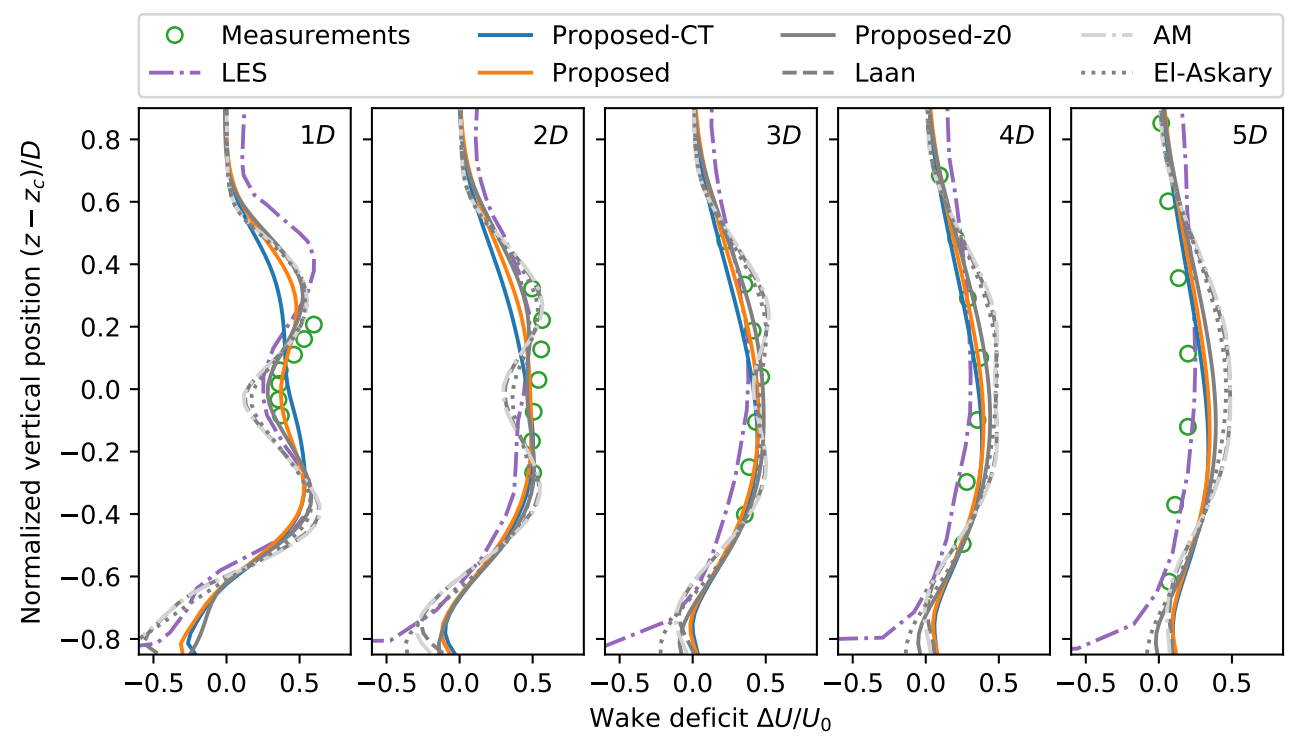

Figure 11. Vertical wake deficit profile of a NTK500/41 wind turbine: $L=29 \mathrm{~m}, \Delta U$ is the velocity deficit in the wake and $U_{0}$ is the inlet velocity.

\subsection{Case 2: Wakes of Danwin $180 \mathrm{~kW}$ Wind Turbines}

The numerical results of the turbulence models based on MOST and the proposed model based on the new similarity functions are compared with the experimental data reported by Magnusson et al. [6] in Figs. 12-14. Comparison is made at distances 4.2, 6.1 and 9.6 D downstream of the turbine. 
Since the detailed rotor geometry of the Danwin $180 \mathrm{~kW}$ wind turbine is not available, the actuator disk model based on the thrust coefficient is applied.

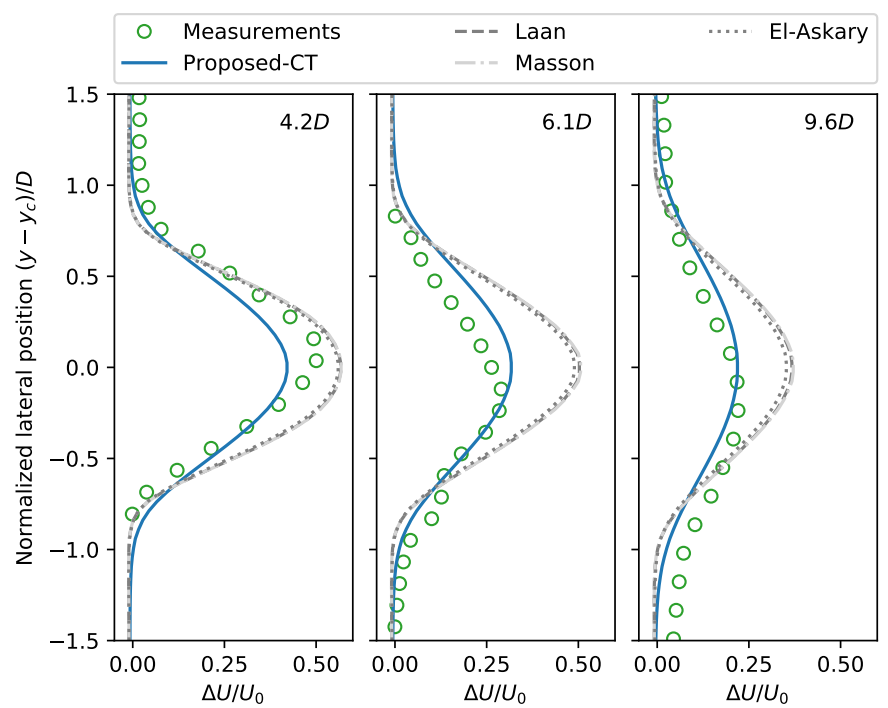

Figure 12. Lateral wake deficit of Danwin $180 \mathrm{~kW}$ wind turbines at hub height: $L=35 \mathrm{~m}, \Delta U$ is the velocity deficit in wake and $U_{0}$ is the inlet velocity and all turbulence models are combined with AD based on thrust coefficient.

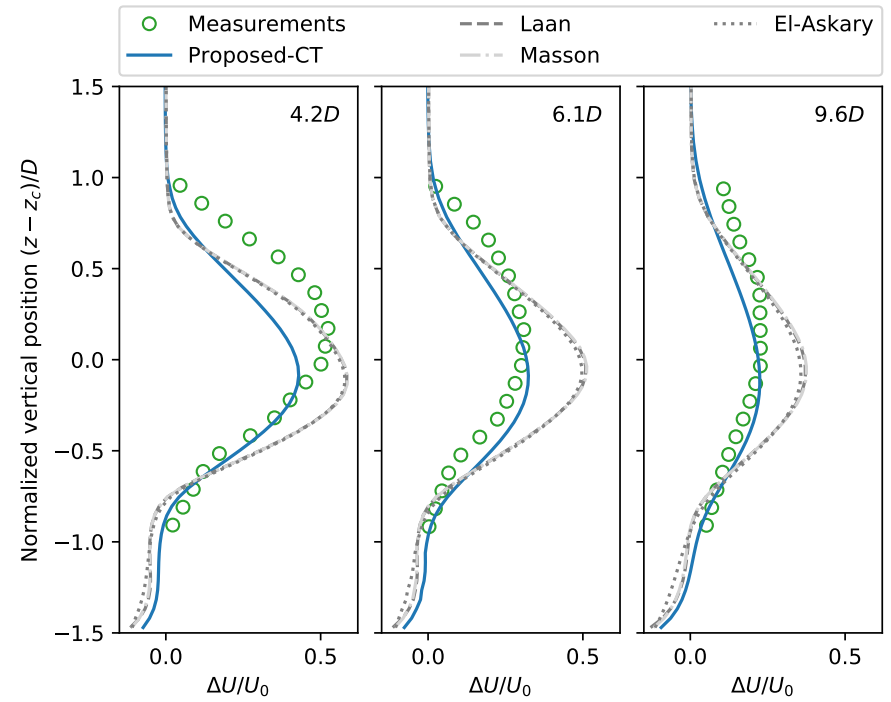

Figure 13. Vertical wake deficit of Danwin $180 \mathrm{~kW}$ wind turbines: $L=35 \mathrm{~m}, \Delta U$ is the velocity deficit in the wake and $U_{0}$ is the inlet velocity and all turbulence models are combined with AD based on thrust coefficient.

Fig. 12-13 demonstrates the change of the wake deficit with the distance downstream of the turbine in both lateral and vertical directions. From this figure, it can be depicted that there is no significant difference between AM, El-Askary and Laan models at all downstream positions. As compared to measurements, these models that are based on MOST, predict larger wake deficits and the wake deficit can be overestimated by 0.25 at $6.1 \mathrm{D}$ downstream of the rotor. Introducing the new similarity functions into the Laan model, improves the wake prediction at $6.1 \mathrm{D}$ and $9.6 \mathrm{D}$ downstream, as compared with the models based on MOST. It should be noted that the measured wake center or the position of the maximum wake deficit, shifts about $0.2 \mathrm{D}$ in both vertical and lateral directions at $6.1 \mathrm{D}$ 
and 9.6 D downstream of the rotor. This shift of the wake center was also observed in full-scale-turbine wakes with scanning-LiDAR measurements from the Crop Wind Energy eXperiment (CWEX) in Iowa [43]. The stretching of the wake structures can be attributed to the strong wind veer associated with stable conditions [6,43]. As wind veer is a direct result of Coriolis effects caused by the Earth's rotation and the Coriolis force is not modeled in this work, all the test models thus fail to capture this stretching of the wake structures.

Fig. 14 shows the distribution of the normalized added turbulence $\sigma_{u}^{2} / \sigma_{u 0}^{2}-1$ in the lateral direction at hub height where $\sigma_{u}$ and $\sigma_{u 0}$ is the standard derivations of wind speed in wakes and in the atmosphere, respectively. A duel-peak pattern (lateral distance $y-y_{c}= \pm 0.5 D$ ) is detected experimentally at $4 D$ downstream of the rotor as a result of rotor tip vortices and high shear production of turbulent kinetic energy caused by strong velocity gradients at wake boundary [3,4]. All test models capture this duel-peak pattern with lower values: the measured peaks reduce by 3 in the simulations using models based on MOST and reduce by 5 in the simulations using the proposed model. This underestimation of the added turbulence may be due to representing the turbine rotor with actuator disk instead of the actual rotor geometry. To note, the duel-peak pattern predicted by the models based on MOST can be observed at distance downstream of the rotor up to $9.6 \mathrm{D}$, which becomes indistinctly at 6.1 D and 9.6 D downstream of the rotor for both measurements and the simulations using the proposed model. As compared to measurements, the models based on MOST also overestimate the normalized added turbulence which is improved by the proposed model at $6.1 \mathrm{D}$ and $9.6 \mathrm{D}$ downstream of the rotor.

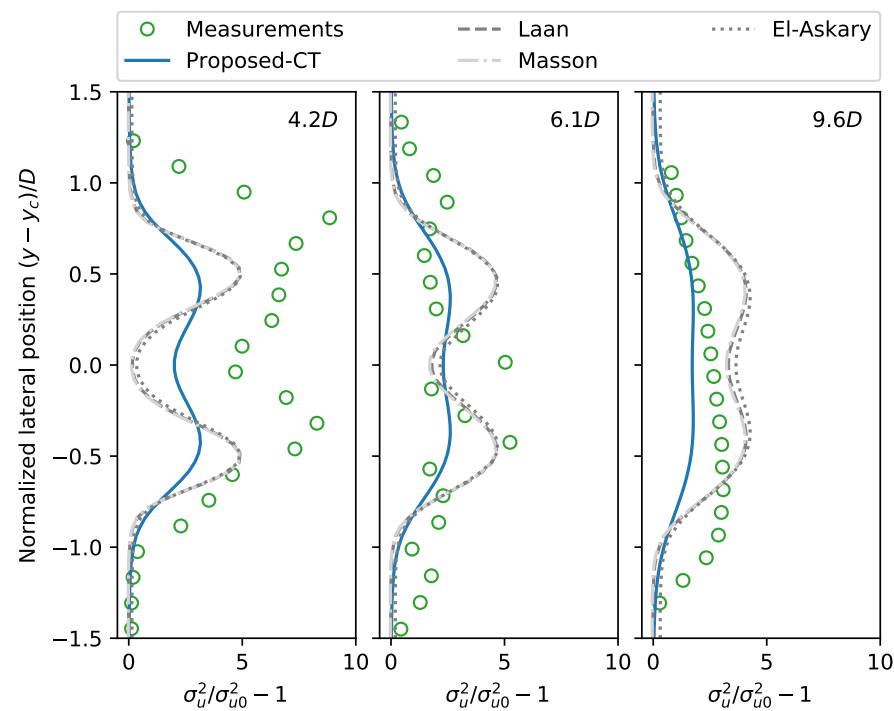

Figure 14. Lateral added turbulence in wakes of Danwin $180 \mathrm{~kW}$ wind turbines at hub height: $L=35$ $\mathrm{m}, \sigma_{u}$ and $\sigma_{u 0}$ is the standard derivations of wind speed in wakes and in the atmosphere. All turbulence models are combined with AD based on thrust coefficient.

\subsection{Model Assessment}

In this section, we adopt the root-mean-square error (RSME) to analyze the accuracy of the CFD predictions using the various turbulence models [44]:

$$
\mathrm{RSME}=\sqrt{\frac{\sum_{1}^{n}\left(y_{\mathrm{CFD}}-y_{\mathrm{EXP}}\right)^{2}}{n}}
$$

where $n$ is the number of measurement points in the evaluation; $y_{\mathrm{EXP}}$ is the experimental data and $y_{C F D}$ is the simulated values from CFD.

The RMSE analysis of the lateral wake deficit is presented in Figs. 15-16. Over a range of 0.025-0.2, the lower the RSME value, the better the behavior of the numerical model. According to Figs. 15, it 
can be depicted that the proposed model either based on BEM in Case 1 or either based on the thrust coefficient in Case 2, has the best performance with the lowest RMSE values (except for 5D in Case 1), especially for the downstream distance to rotor $x-x_{c}>2 D$. AM, El-Askary and Laan model have similar performance in the wake prediction, but El-Askary model has lower RMSE values among the various turbulence models based on MOST. As compared to AM and Laan model, the El-Askary model could reduce RSME of the lateral wake deficit by 0.025 in Case 1 and by 0.015 in Case 2. However, this improvement is smaller than that of the proposed model: introducing the new similarity functions into the Laan model could reduce the RSME of the lateral wake deficit by 0.05 averagely and even by 0.1 for the maximum at $6 D$ downstream of the rotor (Fig. 15). The LES approach presented in Machefaux et al. [1], predicts a decreased RSME of the lateral wake deficit with the distance downstream of the rotor. To note, all the test models except for the LES approach, have poor performance in predicting wake structure at $5 D$ downstream of the rotor. This could be a result of the downslope terrain or uncertainties due to LiDAR measurements [1].

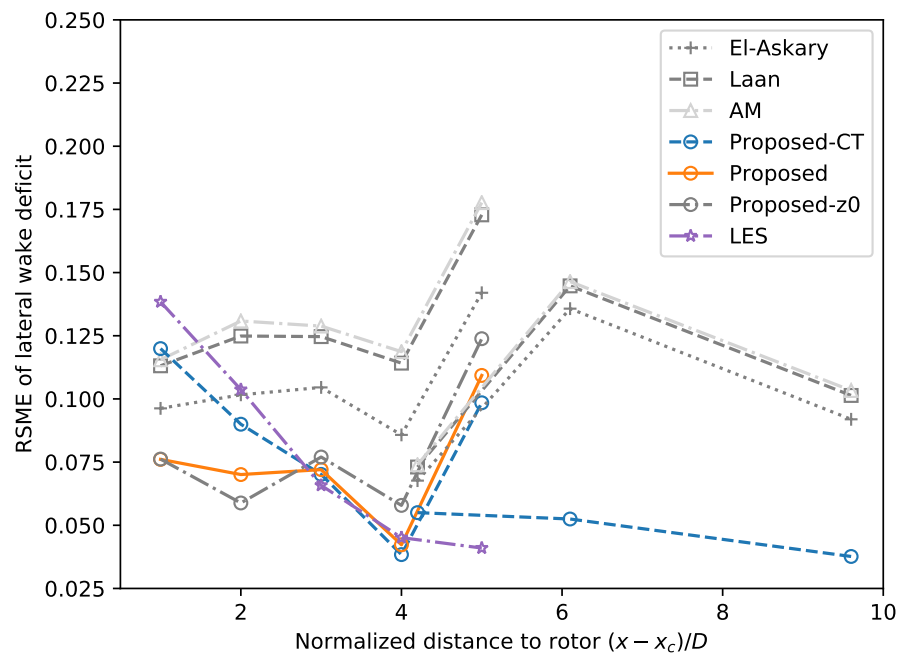

Figure 15. RSME of the wake deficit at hub height under stable conditions: Proposed-CT stands for proposed model using AD based on the thrust coefficient and Proposed-z0 stands for the proposed model imposing a reduced hub-height turbulence intensity. 


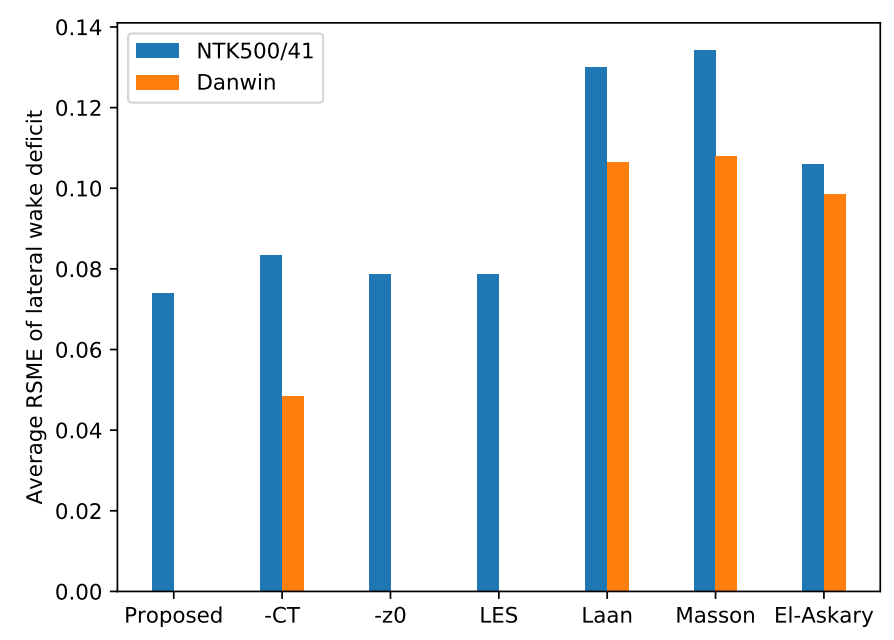

Figure 16. Average of RSME of the wake deficit at hub height under stable conditions: "-CT" stands for proposed model using AD based on the thrust coefficient and "-z0" stands for the proposed model imposing a reduced hub-height turbulence intensity.

Using BEM to distribute force through the rotor significantly improves near wake prediction at $1 D-2 D$ downstream of the rotor in Case 1: the RSME of lateral wake deficit predicted by the proposed model using BEM approach is lower $0.025-0.05$ than the proposed model using $C_{T}$ approach. However, it should be noted that the proposed model using $C_{T}$ approach has a similar performance in the wake prediction with that using BEM approach at $3 D-5 D$ downstream of the rotor. This suggests that the distribution of the momentum source through the rotor only affects near wake structures up to $2 D$ and that one can expect similar accuracy of wake prediction for far wake regardless of which method to distribute the force through the rotor.

Figs. 15-16 also show that imposing the equivalent ambient turbulence intensity in the proposed model enhance wake effects and cannot predict the large wake deficit predicted by the models based on MOST. This indicates that the side effects of the breakdown of MOST on wake modeling under stable conditions could be caused by both the underpredicted turbulence intensity and the overestimated wind shear.

The RMSE analysis of the added turbulence is presented in Figs. 17. The RMSE of the added turbulence is observed to increase with the distance downstream the rotor for the models based on MOST and to decrease with the distance downstream the rotor for the proposed model. The increase of the RMSE of the added turbulence with the distance to the rotor for the models based on MOST, suggests a slower wake recovery predicted by the models based on MOST. In all distances, the RMSE of the added turbulence predicted by the proposed model is lower than that of models based on MOST and the difference in RMSE increases from 0.5 at $4 D$ to about 2 at 9.6D. This indicates that introducing the new similarity functions into the Laan model can eliminate the side effects of the breakdown of MOST on wake modeling. 


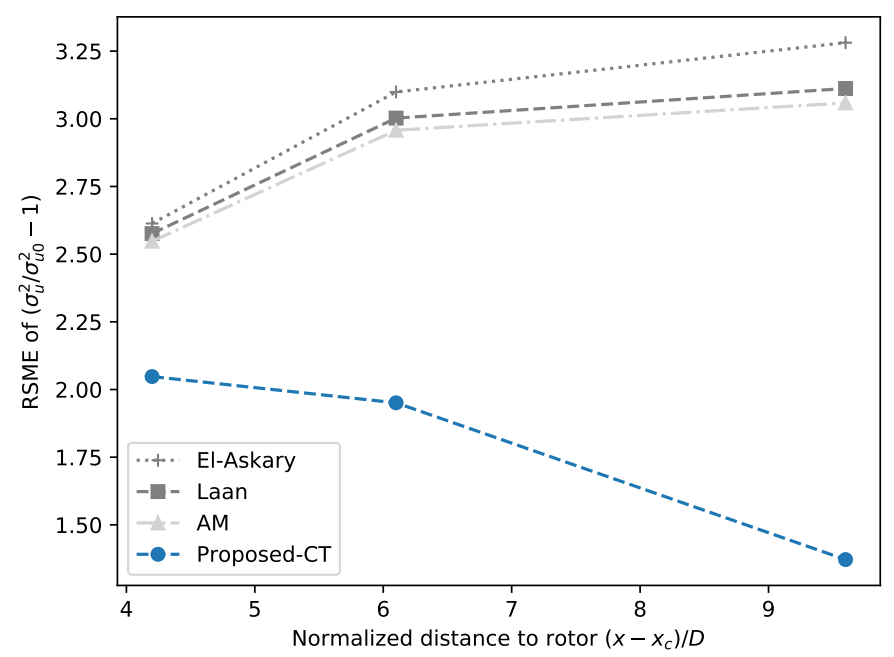

Figure 17. RSME of the lateral added turbulence in wakes of Danwin $180 \mathrm{~kW}$ wind turbines at hub height: $L=35 \mathrm{~m}, \sigma_{u}$ and $\sigma_{u 0}$ is the standard derivations of wind speed in wakes and in the atmosphere. All turbulence models are combined with $\mathrm{AD}$ based on thrust coefficient.

\section{Conclusions}

In the present paper, the breakdown of MOST is investigated experimentally and its side effects on wake modeling through numerical simulations are also investigated. New similarity functions based on a field measurement in a wind farm are proposed and applied in a turbulence model to eliminate the side effects of the breakdown of MOST on wake modeling. Wake simulations of two types of turbines under different stability conditions are carried out and compared with measurements from a LiDAR and cup anemometers. The main findings are: (1) Field measurements show that MOST is only valid for $R_{f}<0.25$ and overestimates wind shear for $\zeta>0.1$. The proposed similarity functions can limit the wind shear as the stability increases. (2) Due to the breakdown of MOST for $\zeta>0.1$, the test models based on MOST overestimates wake effects in both wake deficits and added turbulence under stable conditions. (3) The new similarity functions constrain wind shear for $\zeta>0.1$ as compared with MOST. And introducing the new similarity functions into the Laan model improves the wake prediction under stable conditions. (4) By distributing the blade force through the rotor, momentum effects of the rotor to the atmospheric boundary layer are simulated in a more detail way than the uniformly distributed blade force applied based on the thrust coefficient. This enables the proposed model to capture the double-bell near-wake shape. (5) Imposing the equivalent ambient turbulence intensity in the proposed model enhance wake effects and cannot predict the large wake deficit predicted by the models based on MOST. This indicates that the side effects of the breakdown of MOST on wake modeling under stable conditions could be related to the underpredicted turbulence intensity and the overestimated wind shear.

Author Contributions: Data curation, Xing Xing Han; Formal analysis, Xing Xing Han; Methodology, Xing Xing Han; Project administration, De You Liu; Software, Xing Xing Han and Lin Min Li; Supervision, De You Liu; Validation, Xing Xing Han and Fei Fei Xue; Writing - original draft, Xing Xing Han; Writing - review \& editing, Chang $\mathrm{Xu}$ and Wen Zhong Shen.

Funding: This research was funded by the Joint Funds of the National Natural Science Foundation of China grant number U1865101, Jiangsu provincial science and Technology Department grant number BZ2018007, the Ministry of Science and Technology of China grant number 2014DFG62530, the Danish Energy Agency grant number 64013-0405.

Conflicts of Interest: The authors declare no conflict of interest.

\section{Abbreviations}


438

$\begin{array}{ll}\text { MOST } & \text { Moin-Obukhov Similarity Theory } \\ \text { BEM } & \text { Blade Element Theory } \\ \text { RANS } & \text { Reynolds-averaged Navier-Stokes Equations } \\ \text { LES } & \text { Large Eddy Simulation } \\ \text { AD } & \text { Actuator Disk } \\ \text { AL } & \text { Actuator Line } \\ \text { TKE } & \text { Turbulence Kinetic Energy } \\ \text { LiDAR } & \text { Light Detection and Ranging } \\ \text { CFD } & \text { Computational Fluid Dynamics } \\ \text { OpenFOAM } & \text { Open-source Field Operation And Manipulation }\end{array}$

\section{References}

1. Machefaux, E.; Larsen, G.C.; Koblitz, T.; Troldborg, N.; Kelly, M.C.; Chougule, A.; Hansen, K.S.; Rodrigo, J.S. An experimental and numerical study of the atmospheric stability impact on wind turbine wakes. Wind Energy 2016, 19, 1785-1805.

2. Han, X.; Liu, D.; Xu, C.; Shen, W.Z. Atmospheric stability and topography effects on wind turbine performance and wake properties in complex terrain. Renewable energy 2018, 126, 640-651.

3. Chamorro, L.P.; Porté-Agel, F. Effects of Thermal Stability and Incoming Boundary-Layer Flow Characteristics on Wind-Turbine Wakes: A Wind-Tunnel Study. Boundary-Layer Meteorology 2010, 136, 515-533. doi:10.1007/s10546-010-9512-1.

4. Zhang, W.; Markfort, C.D.; Porté-Agel, F. Wind-turbine wakes in a convective boundary layer: A wind-tunnel study. Boundary-layer meteorology 2013, 146, 161-179.

5. Hancock, P.; Zhang, S.; Pascheke, F.; Hayden, P. Wind tunnel simulation of a wind turbine wake in neutral, stable and unstable wind flow. Journal of Physics: Conference Series. IOP Publishing, 2014, Vol. 555, p. 012047.

6. Magnusson, M.; Smedman, A.S. Influence of atmospheric stability on wind turbine wakes. Wind Engineering 1994, pp. 139-152.

7. Iungo, G.V.; Porté-Agel, F. Volumetric lidar scanning of wind turbine wakes under convective and neutral atmospheric stability regimes. Journal of Atmospheric and Oceanic Technology 2014, 31, 2035-2048.

8. Menke, R.; Vasiljević, N.; Hansen, K.S.; Hahmann, A.N.; Mann, J. Does the wind turbine wake follow the topography? A multi-lidar study in complex terrain. Wind Energy Science 2018, 3, 681-691.

9. Wu, Y.T.; Porté-Agel, F. Atmospheric turbulence effects on wind-turbine wakes: An LES study. energies 2012, 5, 5340-5362.

10. Xie, S.; Archer, C.L. A numerical study of wind-turbine wakes for three atmospheric stability conditions. Boundary-Layer Meteorology 2017, 165, 87-112.

11. Wu, Y.T.; Porté-Agel, F. Large-eddy simulation of wind-turbine wakes: evaluation of turbine parametrisations. Boundary-layer meteorology 2011, 138, 345-366.

12. A Numerical Investigation of Nacelle Anemometry for a HAWT Using Actuator Disc and Line Models in CFX. Renewable Energy 2012, 48, 72-84. doi:10.1016/j.renene.2012.04.004.

13. Prospathopoulos, J.M.; Politis, E.S.; Rados, K.G.; Chaviaropoulos, P.K. Evaluation of the Effects of Turbulence Model Enhancements on Wind Turbine Wake Predictions. Wind Energy 2011, 14, 285-300. doi:10.1002/we.419.

14. El-Askary, W.; Sakr, I.; AbdelSalam, A.M.; Abuhegazy, M. Modeling of Wind Turbine Wakes under Thermally-Stratified Atmospheric Boundary Layer. Journal of Wind Engineering and Industrial Aerodynamics 2017, 160, 1-15. 00000, doi:10.1016/j.jweia.2016.11.001.

15. Alinot, C.; Masson, C. $k$ - varepsilon Model for the Atmospheric Boundary Layer Under Various Thermal Stratifications. Journal of Solar Energy Engineering 2005, 127, 438-443.

16. van der Laan, M.P.; Kelly, M.C.; Sørensen, N.N. A new k-epsilon model consistent with Monin-Obukhov similarity theory. Wind Energy 2017, 20, 479-489. 
17. Grachev, A.A.; Andreas, E.L.; Fairall, C.W.; Guest, P.S.; Persson, P.O.G. The critical Richardson number and limits of applicability of local similarity theory in the stable boundary layer. Boundary-layer meteorology 2013, 147, 51-82.

18. Van Der Avoird, E.; Duynkerke, P.G. Turbulence in a katabatic flow. Boundary-Layer Meteorology 1999, 92,37-63.

19. Foken, T. 50 years of the Monin-Obukhov similarity theory. Boundary-Layer Meteorology 2006, 119, 431-447.

20. Schlichting, H.; Gersten, K. Boundary-layer theory; Springer, 2016.

21. Launder, B.E.; Spalding, D.B. The numerical computation of turbulent flows. Computer Methods in Applied Mechanics and Engineering 1990, 3, 269-289.

22. Businger, J.A.; Wyngaard, J.C.; Izumi, Y.; Bradley, E.F. Flux-profile relationships in the atmospheric surface layer. Journal of the atmospheric Sciences 1971, 28, 181-189.

23. Dyer, A. A review of flux-profile relationships. Boundary-Layer Meteorology 1974, 7, 363-372.

24. Koblitz, T.; Sørensen, N.N.; Bechmann, A.; Sogachev, A. CFD Modeling of Non-Neutral Atmospheric Boundary Layer Conditions; DTU Wind Energy, 2013.

25. Shen, W.Z.; Mikkelsen, R.; Sørensen, J.N.; Bak, C. Tip Loss Corrections for Wind Turbine Computations. Wind Energy 2005, 8, 457-475. doi:10.1002/we.153.

26. Drela, M. XFOIL: An analysis and design system for low Reynolds number airfoils. In Low Reynolds number aerodynamics; Springer, 1989; pp. 1-12.

27. Du, Z.; Selig, M. A 3-D stall-delay model for horizontal axis wind turbine performance prediction. 1998 ASME Wind Energy Symposium, 1998, p. 21.

28. Shen, W.Z.; Zhu, W.J.; Sørensen, J.N. Actuator Line/Navier-Stokes Computations for the MEXICO Rotor: Comparison with Detailed Measurements. Wind Energy 2012, 15, 811-825. doi:10.1002/we.510.

29. Glauert, H. Airplane propellers. In Aerodynamic theory; Springer, 1935; pp. 169-360.

30. El Kasmi, A.; Masson, C. An extended k- $-\varepsilon$ model for turbulent flow through horizontal-axis wind turbines. Journal of Wind Engineering and Industrial Aerodynamics 2008, 96, 103-122.

31. Grachev, A.A.; Andreas, E.L.; Fairall, C.W.; Guest, P.S.; Persson, P.O.G. On the turbulent Prandtl number in the stable atmospheric boundary layer. Boundary-Layer Meteorology 2007, 125, 329-341.

32. Wallace, J.M.; Hobbs, P.V. Atmospheric science: an introductory survey; Vol. 92, Elsevier, 2006.

33. Bak, C.; Fuglsang, P.; Sørensen, N.N.; Madsen, H.A.; Shen, W.Z.; Sørensen, J.N. Airfoil characteristics for wind turbines 1999.

34. Johansen, J.; Sørensen, N.N. Aerofoil characteristics from 3D CFD rotor computations. Wind Energy: An International Journal for Progress and Applications in Wind Power Conversion Technology 2004, 7, 283-294.

35. Vignaroli, A. UniTTe-MC1-Nordtank Measurement Campaign (Turbine and Met Masts) 2016.

36. Magnusson, M. Near-wake behaviour of wind turbines. Journal of Wind Engineering and Industrial Aerodynamics 1999, 80, 147-167.

37. Zhang, X. CFD simulation of neutral ABL flows 2009.

38. Sørensen, J.N.; Mikkelsen, R.F.; Henningson, D.S.; Ivanell, S.; Sarmast, S.; Andersen, S.J. Simulation of wind turbine wakes using the actuator line technique. Philosophical Transactions of the Royal Society A: Mathematical, Physical and Engineering Sciences 2015, 373, 20140071.

39. Chang, C.Y.; Schmidt, J.; Dörenkämper, M.; Stoevesandt, B. A consistent steady state CFD simulation method for stratified atmospheric boundary layer flows. Journal of Wind Engineering and Industrial Aerodynamics 2018, 172, 55-67.

40. Weller, H.G.; Tabor, G.; Jasak, H.; Fureby, C. A tensorial approach to computational continuum mechanics using object-oriented techniques. Computers in physics 1998, 12, 620-631.

41. Sørensen, N.N. General purpose flow solver applied to flow over hills 1995.

42. Menke, R.; Vasiljević, N.; Hansen, K.S.; Hahmann, A.N.; Mann, J. Does the Wind Turbine Wake Follow the Topography? A Multi-Lidar Study in Complex Terrain. Wind Energy Science 2018, 3, 681-691. doi:10.5194/wes-3-681-2018.

43. Bodini, N.; Zardi, D.; Lundquist, J.K. Three-Dimensional Structure of Wind Turbine Wakes as Measured by Scanning Lidar. Atmos. Meas. Tech. 2017, p. 16.

44. Ntinas, G.K.; Shen, X.; Wang, Y.; Zhang, G. Evaluation of CFD turbulence models for simulating external airflow around varied building roof with wind tunnel experiment. Building Simulation. Springer, 2018, Vol. 11, pp. 115-123. 
